MATHEMATICS OF COMPUTATION

Volume 76, Number 260, October 2007, Pages 2241-2248

S 0025-5718(07)02033-9

Article electronically published on May 30, 2007

\title{
NEW TECHNIQUES FOR BOUNDS ON THE TOTAL NUMBER OF PRIME FACTORS OF AN ODD PERFECT NUMBER
}

\author{
KEVIN G. HARE
}

\begin{abstract}
Let $\sigma(n)$ denote the sum of the positive divisors of $n$. We say that $n$ is perfect if $\sigma(n)=2 n$. Currently there are no known odd perfect numbers. It is known that if an odd perfect number exists, then it must be of the form $N=p^{\alpha} \prod_{j=1}^{k} q_{j}^{2 \beta_{j}}$, where $p, q_{1}, \cdots, q_{k}$ are distinct primes and $p \equiv \alpha \equiv 1(\bmod 4)$. Define the total number of prime factors of $N$ as $\Omega(N):=\alpha+2 \sum_{j=1}^{k} \beta_{j}$. Sayers showed that $\Omega(N) \geq 29$. This was later extended by Iannucci and Sorli to show that $\Omega(N) \geq 37$. This was extended by the author to show that $\Omega(N) \geq 47$. Using an idea of Carl Pomerance this paper extends these results. The current new bound is $\Omega(N) \geq 75$.
\end{abstract}

\section{INTRODUCTION}

Here and throughout, $n$ is any natural number, and $N$ is a hypothetical odd perfect number. Let $\sigma(n)$ denote the sum of the positive divisors of $n$. We say that $n$ is perfect if $\sigma(n)=2 n$. It is known that if $\sigma(n)=2 n$ and $n$ is even, then $n=2^{k-1}\left(2^{k}-1\right)$ where $2^{k}-1$ is a Mersenne prime. Currently there are no known odd perfect numbers. First shown by Euler, it is well known that if an odd perfect number exists, then it must be of the form

$$
N=p^{\alpha} \prod_{j=1}^{k} q_{j}^{2 \beta_{j}},
$$

where $p, q_{1}, \cdots, q_{k}$ are distinct primes and $p \equiv \alpha \equiv 1(\bmod 4)$.

Based on (11) we define the total number of prime factors of an odd perfect number as

$$
\Omega(N):=\alpha+2 \sum_{j=1}^{k} \beta_{j},
$$

and we define the total number of distinct prime factors of $N$ as

$$
\omega(N):=1+k .
$$

A number of bounds have been derived for $\Omega(N)$. Cohen showed that $\Omega(N) \geq 23$ 3]. Sayers showed that $\Omega(N) \geq 29$ [13. Iannucci and Sorli showed that $\Omega(N) \geq 37$

Received by the editor July 25, 2005 and, in revised form, October 10, 2005.

2000 Mathematics Subject Classification. Primary 11A25, 11 Y70.

Key words and phrases. Perfect numbers, divisor function, prime numbers.

The research of the author was supported in part by NSERC of Canada. 
TABLE 1. Timing results for algorithm

\begin{tabular}{|l|l|l|}
\hline Result & Time to Prove result & Lines to Prove result \\
\hline$\Omega(N) \geq 69$ & 7 days, 17 hours, 23 minutes, 50 seconds & $25,234,392$ lines \\
$\Omega(N) \geq 71$ & 10 days, 22 hours, 18 minutes, 4 seconds & $37,752,127$ lines \\
$\Omega(N) \geq 73$ & 17 days, 1 hour, 28 minutes, 36 seconds & $56,352,999$ lines \\
$\Omega(N) \geq 75$ & 25 days, 3 hours, 17 minutes, 35 seconds & $83,902,264$ lines \\
\hline
\end{tabular}

[8. The author extended this to give $\Omega(N) \geq 47$ [7. This paper extends this result to give

Theorem 1.1. If $N$ is an odd perfect number, then $\Omega(N) \geq 75$.

In proving these results, the methods of [7] were modified and applied. For an introduction and explanation of the algorithm we refer the interested reader there.

To some extent, with the modification of the algorithm given here, the calculation becomes a matter of bookkeeping. In [7] there was a specific roadblock which prevented any further calculation. This is no longer the case. With enough computational power, we could get to any number, although the amount of time required appears to be exponential, more than doubling every time we increase the bound by 4 (see Table 11). So it is just a matter of how much computer power to dedicate towards the problem. The choice of 75 in the theorem was motiviated by the fact that it was sufficiently large to demonstrate the improvement in the algorithm while still remaining reasonable with respect to computation time.

\section{Definitions AND NOTATION}

For any prime $p$, by $p^{a} \| N$ we mean $p^{a} \mid N$ and $p^{a+1} \nmid N$. By $p^{a} \nVdash N$ we mean either $p^{a+1} \mid N$ or $p^{a} \nmid N$. We define the function $\sigma_{-1}(n)$ as

$$
\sigma_{-1}(n):=\sum_{d \mid n} d^{-1}=\frac{\sigma(n)}{n} .
$$

A number of simple results concerning $\sigma_{-1}(n)$ are summarized below.

Lemma 2.1. Let $n$ be any natural number. Then

- $\sigma_{-1}(n)$ is a multiplicative function, i.e. if $(n, m)=1$, then $\sigma_{-1}(n \cdot m)=$ $\sigma_{-1}(n) \sigma_{-1}(m)$

- $\sigma_{-1}(n)>1$ for all $n>1$,

- $\sigma_{-1}(n)=2$ if and only if $n$ is perfect,

- $\frac{p+1}{p} \leq \sigma_{-1}\left(p^{a}\right)<\sigma_{-1}\left(p^{a+1}\right)<\frac{p}{p-1}$ for all primes $p$ and integers $a \geq 1$.

There are a number of useful results concerning $\omega(N)$, the total number of distinct prime factors.

Lemma 2.2. Let $N$ be an odd perfect number. Then

- $\omega(N) \geq 8[2,5$.

- If $3 \nmid N$, then $\omega(N) \geq 11$ 6, 9].

- If $3 \nmid N$ and $5 \nmid N$, then $\omega(N) \geq 15$ [11.

- If $3 \nmid N, 5 \nmid N$ and $7 \nmid N$, then $\omega(N) \geq 27$ [11].

- If $3 \nmid N, 5 \nmid N, 7 \nmid N$, and $11 \nmid N$, then $\omega(N) \geq 41$ and hence $\Omega(N) \geq 81$. 
The last result is probably not the tightest possible, but it is sufficient for our purposes. It follows immediately by noticing that the product $\prod \frac{p_{i}}{p_{i}-1}$ for the first 40 primes strictly greater than 11 is less than 2 . So, for our purposes it suffices to assume that $\Omega(N) \leq 73$ and show as a result that $3 \nmid N, 5 \nmid N, 7 \nmid N$ and $11 \nmid N$, which gives the desired contradiction.

This last result is well known in the literature. We use the version given in [4].

Lemma 2.3 (Cohen, Sorli [4]). Let

$$
N=p_{1}^{\alpha_{1}} p_{2}^{\alpha_{2}} \cdots p_{k}^{\alpha_{k}} q_{1}^{\beta_{1}} q_{2}^{\beta_{2}} \cdots q_{r}^{\beta_{r}}
$$

be an odd perfect number, where the $p_{i}$ and $q_{j}$ are distinct primes with the $p_{i}$ and $\alpha_{i}$ known. Define

$$
S:=\sigma_{-1}\left(p_{1}^{\alpha_{1}} \cdots p_{k}^{\alpha_{k}}\right)
$$

Then we have

$$
\min q_{i}<\frac{2+S(r-1)}{2-S} ;
$$

moreover, if $r \geq 3$ we have the tighter bound

$$
\min q_{i}<\frac{8+2 S^{2}(r-1)}{4-S^{2}}
$$

Furthermore we have the lower bound

$$
\min q_{i} \geq \max \left(3, \frac{S}{2-S}\right) .
$$

Proof. Taking $h=\sigma_{-1}, \mu=1, h(\lambda)=S$ and $w=r$, this follows directly from equations $(2.3),(4.1)$ and $(2.4)$ of [4].

Example 2.4. Assume that $N=3^{2} \cdot 13^{4} \cdot 30941 \cdot q_{1}^{\beta_{1}} \cdot q_{2}^{\beta_{2}}$ is an odd perfect number. Then by Lemma 2.3 we can assume that $3.5962<q_{1}<8.1924$. So in, particular we can assume that $q_{1}=5$ or 7 .

\section{The Algorithm And PROOF of TheOREM 1.1}

Suppose $N=p^{\alpha} \prod q_{i}^{2 \beta_{i}}$, as before. To prove that $\Omega(N) \geq K$, we assume that $\Omega(N)=\alpha+\sum 2 \beta_{i} \leq K-2$ and obtain a contradiction for every combination of $\alpha$ and $\beta_{i}$. As mentioned above, it suffices to assume that $\Omega(N) \leq 73$ and show as a result that $3 \nmid N, 5 \nmid N, 7 \nmid N$ and $11 \nmid N$ to obtain our contradiction.

There are two main modifications to the algorithm in [7]. First, as opposed to doing every individual case of $\left[\alpha, \beta_{1}, \cdots, \beta_{k}\right]$ where $\Omega(N)=\alpha+2 \sum \beta_{i}$, we combine them into one test. For example, if we want to prove $3 \nmid N$, where $\Omega(N) \leq 57$, we look at the possibilities $3^{2}\left\|N, 3^{4}\right\| N, \cdots, 3^{56} \| N$, and recurse. (Actually, for any power $\beta \geq 46$, we have that $3^{\beta} \| N$ will give rise to a contradiction as $\omega(N) \geq 8$.) This was done because the original method of looking at every possible partition of $N$ caused a large amount of duplicated effort in the automated proofs. By combining these tests together we speed up the calculation and significantly reduce the storage space requirements. 
It should be pointed out that this modification introduced a new means of obtaining a contradiction, which in [7] was taken care of in the choice of partitions. This is listed as contradiction (5D) below.

The second modification to the algorithm is the use of Lemma 2.3. If the algorithm finds itself in a situation where previously it could not continue, because it did not know the factorization of some very large number, we then compute $\frac{2+S(r-1)}{2-S}$ or $\frac{8+2 S^{2}(r-1)}{4-S^{2}}$ as appropriate, from Lemma 2.3. If this upper bound is reasonably small, then we run though all of the possibilities of $q_{i}$ prime less than this upper bound (and greater than the lower bound), as a means of continuing the calculation. Here "small" was defined as anything less than 100000. This was sufficient for these calculations. It should also be noted that the primes are checked in order. After proving that a particular prime (say $p$ ) causes a contradiction, it is assumed that it cannot occur as a factor for the next cases being checked, within the same sub-branch. (For example, with an abuse of notation, we assume $p^{0} \| N$, and would arrive at contradiction (1) if a factor of $p$ occurs.)

There are five contradictions that we test for. The first four are from the original algorithm, or the equivalent variation needed to combine all tests into one test. The last contradiction is commented on above.

(1) Excess of a given prime:

By assuming $p^{k} \| N$ we derive the contradiction that $p^{k+1} \mid N$. This is denoted in the output by "xs=p" where $p$ is the prime in question.

For example, if we wish to show that $269 \nVdash N$ assuming that $3^{2} \| N$, we first assume that $269 \| N$. Then we see that $3^{3}$ and 5 must divide $N$ (the factors of $\sigma(269)$ ), which contradicts $3^{2} \| N$. In this case, this would be denoted in the output by " $\mathrm{xs}=3$ ".

(2) Excess of the number of primes:

We have more primes than we are allowed, given the restrictions on $\Omega(N)$ and the fact that only one prime can have an exponent of 1 . This is denoted in the output by "xs=prime". Incompletely factored numbers are counted as contributing two primes, even though this may be too low. Incompletely factored numbers are known not to be perfect powers. Furthermore, incompletely factored numbers are checked to ensure that they are co-prime with each other, as well as other primes within the relevant branch.

For example, if we wish to show that $26881^{14} \nVdash N$ when $\Omega(N) \leq 19$, we would start with the assumption that $26881^{14} \| N$. This would imply that $3,5,31,43,3368729516337631,6717545999551,5601667,8265157321$, 18691, 145861 and 1801 must all divide $N$, as they are the factors of $\sigma\left(26881^{14}\right)$. We see that the factorization of $N$ that would maximize the number of prime factors would be $N=26881^{14} \cdot p_{1} \cdot p_{2}^{2} \cdot p_{3}^{2}$. As this has at most 3 primes unassigned, and there are 11 unassigned prime factors of $\sigma\left(26881^{14}\right)$, we get a contradiction.

(3) Partition cannot be satisfied:

The factors that must divide $N$, along with their powers, cannot satisfy the partition. In the original algorithm there were a number of different ways that this contradiction could occur. Given that in this implementation there are not specific exponent bounds assigned before starting to recurse, this can only occur in one way. This is if, of the remaining primes, one of 
them has to be the special prime (with an exponent $a \equiv 1(\bmod 4))$, and of all of the remaining primes, all of them are such that $p \not \equiv 1(\bmod 4)$. This is denoted in the output by "exponent bounds exceeded".

For example, if we wished to show that $3^{10} \sharp N$ when $\Omega(N) \leq 13$, we would start by assuming that $3^{10} \| N$. This implies that 23 and 3851 both must divide $N$ (the two factors of $\sigma\left(3^{10}\right)$ ). At this point, as $\Omega(N) \leq 13$, we must have one of these two primes being the special prime to get either $N=3^{10} \cdot 23 \cdot 3851^{2}$ or $N=3^{10} \cdot 23^{2} \cdot 3851$. But we notice that $23 \equiv 3851 \equiv 3$ (mod 4); hence we see that neither of 23 or 3851 could be the special prime, hence a contradiction.

(4) Excess of $\sigma_{-1}$ :

A lower bound for $\sigma_{-1}(N)$ using known factors gives $\sigma_{-1}(N)>2$. This is denoted in the code by " $\mathrm{S}=$ number", giving a floating point approximation for a lower bound of $\sigma_{-1}(N)$. (The code uses exact rational arithmetic to check the inequality.)

For example, if we wished to show that $90089 \nVdash N$ we would start by assuming that $90089 \| N$. This implies that $3^{2}, 5,7,11$ and 13 all divide $N$ (the factors of $\sigma(90089)$ ). This implies that

$$
\begin{aligned}
\sigma_{-1}(N) & \geq \frac{\sigma\left(3^{2}\right)}{3^{2}} \times \frac{\sigma(5)}{5} \times \frac{\sigma(7)}{7} \times \frac{\sigma(11)}{11} \times \frac{\sigma(13)}{13} \times \frac{\sigma(90089)}{90089} \\
& \approx 2.327298560 \\
& >2
\end{aligned}
$$

which is the desired contradiction. This is denoted in the output as "S=2.327298560".

(5) $\omega$-bound exceeded:

Given the choices of primes and exponents made so far, it is not possible to satisfy Lemma 2.2.

For example, if we were trying to prove that $3^{46} \nVdash N$ where $\Omega(N) \leq 57$ we would start by assuming that $3^{46} \| N$. We notice that $\omega(N)$ is maximized by writing $N=3^{46} \cdot p_{1} \cdot p_{2}^{2} \cdot p_{3}^{2} \cdot p_{4}^{2} \cdot p_{5}^{2}$, and hence $\omega(N) \leq 7$. This contradicts $\omega(N) \geq 8$ from Lemma 2.2. This is denoted as "violate omega bound".

In Table 2 an example of running this code is given, as well as the five possible exceptions. These exceptions are indicated by the numbers (1) through (5) on the left hand side of the page. The use of Lemma 2.3 is indicated with a star, $(*)$, on the left hand side of the page. Minor formatting has been done to the output, to avoid lines with more than 80 characters. (The output of the actual code would have put the line starting with "It would be nice to know" all on one line.)

As was done in [7, numbers were factored using the ifactor command in MAPLE, with the easy option specified. If easy factors were not found, then the number was checked in a hints database (which currently contains over 700 completely, or partially factored numbers). 
TABLE 2. Parts of the proof that $\Omega(N) \geq 75$

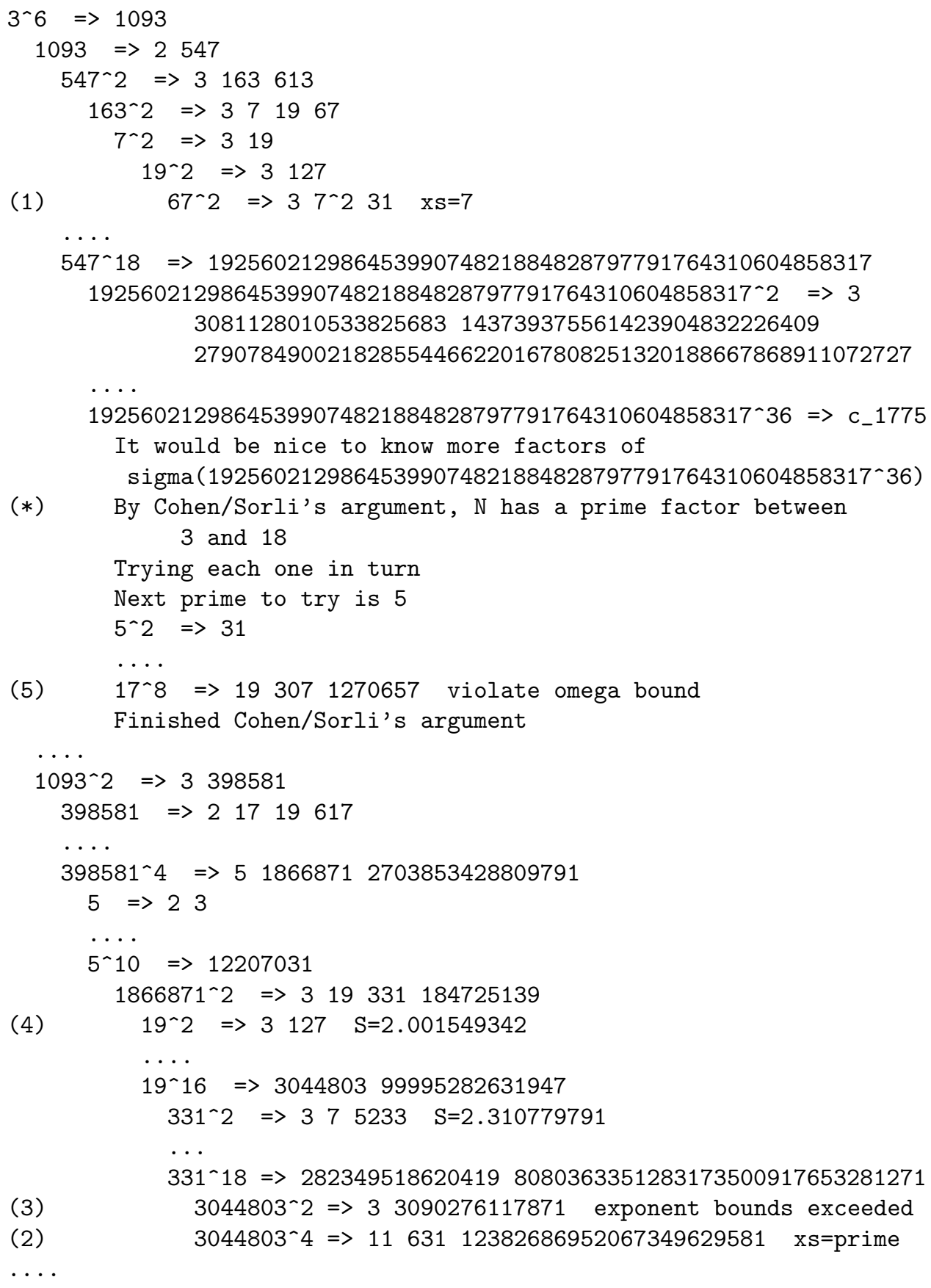




\section{Comments And ACKNOWLEDGments}

In [7] it was said that there were three numbers that needed to be factored, without which the algorithm in [7] could not be continued. Using the methods of Carl Pomerance, this problem was avoided. At this point, the calculation was terminated not by a particular obstruction, but instead because 75 was esthetically pleasing. Further, 75 was sufficiently large to demonstrate the improvement in the algorithm while still remaining reasonable with respect to computation time. The purpose of this paper was to demonstrate a new technique to extend this bound, and not necessarily to extend this bound to the farthest extent possible.

I would like to thank Phil Carmody, Christophe Clavier, Don Leclair, Paul Leyland, Tom Womack, Paul Zimmermann, and the people at http://mersenneforum.org who helped provide factorizations for the numbers

- $\sigma\left(\sigma\left(547^{18}\right)^{16}\right)$, a 789 -digit number, with a factor of:

$$
1520135498523547561326750429418247 .
$$

- $\sigma\left(\sigma\left(3221^{12}\right)^{22}\right)$, a 927-digit number, with a factor of:

$$
46973400441039677515399714233826061,
$$

which I had listed in [7] as obstructions to my calculations. This information was included in the hints database used by the code.

I am also indebted to Carl Pomerance for bringing a weaker form of Lemma 2.3 to my attention [12, and more importantly how this could be applied to improve the existing bound. I would also like to thank the unknown referee for the reference [4, which replaced Lemma 2.3, as well as many other useful suggestions and comments.

Also, I would like to acknowledge William Lipp, the creator of the website www.oddperfect.org, for providing some additional factorizations (this website, when complete, will coordinate an attack to improve the lower bound on odd perfect numbers, of $N \geq 10^{300}$, given by 1 .)

Between acceptance and publication of this paper, the author has become aware of the publication [10]. This would strengthen the first line of Lemma 2.2 to read $\omega(N) \geq 9$.

\section{REFERENCES}

[1] R. P. Brent, G. L. Cohen, and H. J. J. te Riele, Improved techniques for lower bounds for odd perfect numbers, Math. Comp. 57 (1991), no. 196, 857-868. MR.1094940 (92c:11004)

[2] E. Z. Chein, An odd perfect number has at least 8 prime factors, Ph.D. thesis, Pennsylvania State University, 1979.

[3] Graeme L. Cohen, Generalised quasiperfect numbers, Ph.D. thesis, University of New South Wales, 1982.

[4] Graeme L. Cohen and Ronald M. Sorli, On the number of distinct prime factors of an odd perfect number, J. Discrete Algorithms 1 (2003), no. 1, 21-35, Combinatorial algorithms. MR2016472 (2004h:11003)

[5] Peter Hagis, Jr., Outline of a proof that every odd perfect number has at least eight prime factors, Math. Comp. 35 (1980), no. 151, 1027-1032. MR572873 (81k:10004)

[6] Sketch of a proof that an odd perfect number relatively prime to 3 has at least eleven prime factors, Math. Comp. 40 (1983), no. 161, 399-404. MR679455 (85b:11004)

[7] Kevin G. Hare, More on the total number of prime factors of an odd perfect number, Math. Comp. 74 (2005), no. 250, 1003-1008 (electronic). MR:2114661(2005h:11010)

[8] D. E. Iannucci and M. Sorli, On the total number of prime factors of an odd perfect number, Math. Comp. 72 (2003), no. 244, 2077-2084. MR.1986824 (2004b:11008) 
[9] Masao Kishore, Odd perfect numbers not divisible by 3. II, Math. Comp. 40 (1983), no. 161, 405-411. MR679456 (84d:10009)

[10] Pace P. Nielsen, Odd perfect numbers have at least nine distinct factors, Math. Comp. (to appear).

[11] Karl K. Norton, Remarks on the number of factors of an odd perfect number, Acta Arith. 6 (1960/1961), 365-374. MR0147434 (26:4950)

[12] Carl Pomerance, Odd perfect numbers are divisible by at least seven distinct primes, Acta Arith. 25 (1973/74), 265-300. MR0340169(49:4925)

[13] M. Sayers, An improved lower bound for the total number of prime factors of an odd perfect number, Master's thesis, New South Wales Institute of Technology, 1986.

Department of Pure Mathematics, University of Waterloo, Waterloo, Ontario, CANADA, N2L 3G1

E-mail address: kghare@math.uwaterloo.ca 